\title{
One- and Five-Year Risk of Death and Cardiovascular Complications for Hospitalized Patients With Hyperglycemia Without Diagnosed Diabetes: An Observational Study
}

\author{
Meltem Tuna, $\mathrm{PhD}^{1,2}$, Douglas G. Manuel, MD, MSc $c^{1,2,3,4,5,6 \star}$, Carol Bennett, MSc ${ }^{1,2}$, Nadine Lawrence, MSc ${ }^{2,7}$, \\ Carl van Walraven, MD, MSc $c^{1,2,8,9}$, Erin Keely, MD ${ }^{8,9,10}$, Janine Malcolm, MD ${ }^{8,9,10}$, Robert D. Reid, PhD ${ }^{9,11}$, \\ Alan J. Forster, MD, MSc $1,2,7,8,9$
}

\begin{abstract}
IICES@uOttawa, Institute for Clinical Evaluative Sciences, Ottawa, Ontario, Canada; ${ }^{2}$ Clinical Epidemiology Program, Ottawa Hospital Research Institute, Ottawa, Ontario, Canada; ${ }^{3}$ Department of Family Medicine, University of Ottawa, Ottawa, Ontario, Canada; ${ }^{4}$ CT Lamont Primary Health Care Research Centre, University of Ottawa, Ontario, Canada; ${ }^{5}$ Bruyere Research Institute, University of Ottawa, Ontario, Canada; ${ }^{6}$ Department of Epidemiology and Community Medicine, University of Ottawa, Ottawa, Ontario, Canada; ${ }^{7}$ Performance Measurement, The Ottawa Hospital, Ottawa, Ontario, Canada; ${ }^{8}$ Department of Medicine, The Ottawa Hospital, Ottawa, Ontario, Canada; ${ }^{9}$ Department of Medicine, University of Ottawa, Ottawa, Ontario, Canada; ${ }^{10}$ Division of Endocrinology and Metabolism, University of Ottawa, Ottawa, Ontario, Canada; ${ }^{11}$ Minto Prevention and Rehabilitation Centre, University of Ottawa Heart Institute, Ottawa, Ontario, Canada.
\end{abstract}

BACKGROUND: Hyperglycemia is recognized as an important threat to the health of patients, independent of diabetes status. However, no long-term follow-up study of patients with in-hospital elevated glucose without diabetes has been conducted.

OBJECTIVE: To compare 1- and 5-year risk of death and cardiovascular (CV) complications in patients with a diagnosis of diabetes versus those without a diabetes diagnosis

DESIGN: Retrospective cohort study.

METHODS: Risk of all-cause death and CV complications (acute myocardial infarction [AMI], congestive heart failure [CHF], cardiovascular disease [CVD], peripheral vascular disease [PVD], and end-stage renal disease [ESRD]) in those diagnosed with diabetes versus those with different glycemia categories was determined using competing risk models.

SETTING/PATIENTS: All adult patients from a tertiary hospital discharged alive between 1996 and 2008 from any service except psychiatry or obstetrics.
RESULTS: Compared to patients with diagnosed diabetes, patients with peak serum glucose level $>200 \mathrm{mg} / \mathrm{dL}$ had significantly higher 1-year risk (hazard ratio [HR]: 1.31, 95\% confidence interval [Cl]: 1.20-1.43) and 5-year risk (HR: 1.13, 95\% Cl: 1.06-1.22) of death but a decreased 1-year risk of hospitalization for CHF (HR: $0.71,95 \% \mathrm{Cl}$ : 0.620.81), PVD (HR: $0.20,95 \% \mathrm{Cl}: 0.18-0.24)$, or ESRD (HR: $0.73,95 \% \mathrm{Cl}: 0.6-0.89)$. There was no risk difference for AMI (HR: $0.96,95 \% \mathrm{Cl}: 0.78-1.18)$ or CVD (HR: 0.79 , 95\% $\mathrm{Cl}: 0.61-1.0)$.

CONCLUSIONS: Although it is unclear whether hospitalized patients with elevated peak serum glucose have early diabetes or their hyperglycemia reflects hospital stress or another comorbidity, in-hospital hyperglycemia is an important clinical indicator, carrying a higher 1- and 5-year mortality risk than those with diagnosed diabetes. Journal of Hospital Medicine 2014;9:365-371. (C) 2014 Society of Hospital Medicine

The mechanism of stress hyperglycemia differs from diabetic hyperglycemia and can occur independently of diabetes status. ${ }^{8}$ Stress hyperglycemia is characterized by rapid onset of insulin resistance, which normally takes months or years in diabetes mellitus. It develops in association with or because of other stressor such as infection or inflammatory processes. 9

To our knowledge, no long-term follow-up study on hospitalized patients with elevated glucose levels but without a diabetes diagnosis has ever been conducted. In this study, we measured the 1- and 5-year risk of mortality and diabetes-related diseases for hospitalized patients with a diabetes diagnosis compared with patients grouped according to their peak in-hospital serum glucose level. Our primary comparison was between patients with diagnosed diabetes and those with peak serum glucose $>200 \mathrm{mg} / \mathrm{dL}(11.1 \mathrm{mmol} / \mathrm{L})$, but we also examined 4 other categories of peak serum glucose: 140 to $200 \mathrm{mg} / \mathrm{dL}(7.8-11.1 \mathrm{mmol} / \mathrm{L}), 108$ to $140 \mathrm{mg} / \mathrm{dL}$ (6.0-7.8 $\mathrm{mmol} / \mathrm{L})$, and $<108 \mathrm{mg} / \mathrm{dL}(<6.0 \mathrm{mmol} / \mathrm{L})$. 


\section{METHODS}

\section{Study Base}

The study included all adult patients (excluding patients admitted to psychiatry and obstetrics) admitted to The Ottawa Hospital (TOH) between January 1, 1996 and March 31, 2008 and discharged alive. Included patients were 18 years or older and had complete medical record abstracts. $\mathrm{TOH}$ is the primary hospital in Ottawa, Ontario, Canada, and the main tertiary hospital in the Champlain Local Health Integration Network, the public healthcare authority for the Ottawa region. This study was approved by The Ottawa Hospital Research Ethics Board.

\section{Data Sources}

The Ottawa Hospital Data Warehouse (OHDW) is a repository for data from the hospital's patient information systems. These systems include patient registration information, discharge abstract, and laboratory, pharmacy, and radiology results. OHDW was used to determine each patient's age, gender, diagnoses, pharmacy orders, laboratory test results, in-hospital comorbidities, most responsible hospital service, and admission urgency.

This dataset was linked to 3 populationbased administrative datasets and 1 derived cohort. Ontario's Registered Persons Database (RPDB) is a population-based registry containing date of death (if applicable) as well as eligibility status for the provincial universal health insurance program, the Ontario Health Insurance Plan (OHIP). ${ }^{10}$ The OHIP database records billing claims submitted by approximately $95 \%$ of Ontario physicians. Each claim contains a fee code describing the type of service provided and a location denoting where the service had taken place. The Canadian Institute for Health Information Discharge Abstract Database (DAD) records clinical, demographic, and administrative data for all hospital admissions and same-day surgeries for all Ontario acute care hospitals since April 1, 1988. The Ontario Myocardial Infarction Database (OMID) contains records of all patients with a most responsible diagnosis of acute myocardial infarction (AMI) (International Classification of Diseases, 9th Revision [ICD-9] code 410 or International Classification of Diseases, 10th Revision [ICD-10] code I21) identified from the DAD. Details on the creation of the OMID are provided in an earlier study. ${ }^{10}$

\section{Variable Definitions}

We identified the index admission of the TOH cohort in the DAD using a unique encrypted health insurance number, admission and discharge dates, and the institution number. To avoid double counting, patients with the same encrypted health insurance number who were discharged from an institution and admitted to another within 2 days were classified as transfers and counted as 1 hospitalization.
Diabetes and cardiovascular (CV) complications (acute myocardial infarction [AMI], congestive heart failure $[\mathrm{CHF}]$, cardiovascular disease $[\mathrm{CVD}]$, peripheral vascular disease [PVD], and end-stage renal disease [ESRD]) were identified in the discharge abstract as the most responsible diagnosis for the admission as well as postadmission comorbidities. The discharge abstract records diagnostic codes according to ICD-9 (April 1, 1988-March 31, 2002) or ICD-10 (April 1, 2002-March 1, 2009).

Each hospitalization was classified into 1 of 6 mutually exclusive diabetes status categories based on diagnostic codes, serum glucose test results, and pharmacy records. The "diagnosed diabetes" group included hospitalizations with any diagnostic code of diabetes in the discharge abstract or any order for a diabetes medication during hospitalization. Eligible medications included acarbose, acetohexamide, chlorpropamide, glibenclamide, gliclazide, glimepiride, glipizide, glyburide, insulin, metformin, nateglinide, pioglitazone, repaglinide, rosiglitazone, and tolbutamide. A chart validation study showed excellent ascertainment of diabetes status using these methods (correct classification $88.8 \%$; 95\% confidence interval [CI]: 85.791.3 with weighted kappa $=0.89$; 95\% CI: 0.85 0.92). ${ }^{11,12}$ Patients without diagnosed diabetes were classified according to their peak serum glucose value during the index hospitalization: $<108 \mathrm{mg} / \mathrm{dL} \quad(<6.0$ $\mathrm{mmol} / \mathrm{L}), 108$ to $140 \mathrm{mg} / \mathrm{dL}(6.0-7.8 \mathrm{mmol} / \mathrm{L}), 140$ to $200 \mathrm{mg} / \mathrm{dL}(7.8-11.1 \mathrm{mmol} / \mathrm{L}),>200 \mathrm{mg} / \mathrm{dL}(>11.1$ $\mathrm{mmol} / \mathrm{L}$ ); hospitalizations in which glucose levels were not obtained were classified as unknown. These peak serum glucose categories are based on the World Health Organization's definition of diabetes and poor glucose tolerance. ${ }^{12}$

\section{Outcomes}

The primary outcome was all-cause postdischarge mortality determined by linking the index admission to the RPDB. Secondary outcomes were CV complications, including: AMI (determined by linking to the OMID); hospitalization for CHF (determined by linking to the DAD for primary diagnosis of $425,428,514,518.4$ or I50, I42.0, I42.6, I42.7, I42.8, I42.9, I43, J81), CVD (determined by linking to the DAD for primary diagnosis of $430,431,432,434,436$ or I60, I61, I62, I63, I64, G46), PVD (determined by primary diagnosis of 96.11 , 96.12, 96.13, 96.14, 96.15 [excluded if in conjunction with $170,171,213,730,740-759,800-900,901-904$, 940-950], 50.18, 51.25, 51.29 [excluded if in conjunction with 414.1, 441, 44] or 1VC93, 1VG93, 1VQ93, 1WA93, 1WE93, 1WJ93, 1WL93, 1WM93 [excluded if in conjunction with C40, C41, C46.1, C47, C49, D160, M46.2, M86, M87, M89.6, M90.0-M90.5, Q00, Q38Q40, S02.0, S09.0, S04.0, S15, S25, T26), and 1KG50, 1KG57, 1KG76, 1KG35HAC1, 1KG35HHC1 [excluded if in conjunction with I60, I67.1, I71, I72, 177.0, 
179.0]), and ESRD (determined by 403.9, 404.9,584, $585,586,788.5$ or I12, I13, N17, N18, N19, R34).

\section{Analysis}

We identified all encounters of cohort patients in any Ontario acute-care hospital within 5 years following the index discharge. Patients not covered by provincial health insurance (OHIP) were excluded.

We first measured crude mortality and morbidity rates by patient category (diagnosed diabetes and glucose levels). Next, we compared the unadjusted outcomes and baseline characteristics (age, sex, previous inpatient and emergency admissions, and disease at admission) between groups using a $\chi^{2}$ test for categorical variables and analysis of variance for continuous variables.

Due to the violation of proportional hazards, we used the Weibull accelerated failure time model to calculate the hazard of death associated with diabetes status or serum glucose level. Consistency of the probability plots confirmed appropriateness of using the Weibull function. Competing risk is defined as a type of failure that prevents the observation of the event of interest or fundamentally alters the probability of its occurrence. ${ }^{13,14}$ In the comorbidity analyses, death is a competing risk for all other outcomes. We calculated the multivariate competing risk hazard for each outcome of interest except death. Each model was adjusted for potential confounders: baseline risk of in-hospital mortality, common comorbidities, most responsible hospital service, and the number of previous inpatient admissions and emergency department visits to $\mathrm{TOH}$ in the previous 6 months. The probability of dying during the admission was calculated using the Escobar model, which predicts the in-hospital probability of dying using data available at the time of admission to the hospital. ${ }^{15}$ The Escobar model has been validated in the study population. ${ }^{16}$ The baseline probability of dying was based on age, sex, acuity of admission, primary condition, Charlson comorbidity score ${ }^{17}$ and the laboratory-based acute physiology score. ${ }^{15}$ The Charlson comorbidity score was calculated using weights from Schneeweiss et al. ${ }^{17}$ Kaplan-Meier survival curves were created for both adjusted and unadjusted models. Death was used as the main censoring variable when analyzing nonfatal outcomes at 1- and 5-year follow-up.

Adjusted models were constructed using a stepwise selection technique and compared with Akaike and Bayesian information criteria values. For all tests, significance was defined as a $P$ value of 0.05 or less.

We recognize that the cohort may contain more than 1 index hospitalization per patient. However, repeated analyses using only the first or last encounter per patient produced nearly identical hazard ratios (HRs) and CIs.

\section{RESULTS}

Between January 1, 1996 and March 31, 2008, 194,641 nonpsychiatric and nonobstetric adults were admitted to TOH. Seventeen patients were excluded because they were ineligible for healthcare coverage and had no encounter with the Ontario healthcare system following discharge from hospital, and 11,175 of the admissions ended in death. The final cohort consisted of 114,764 unique individuals representing 183,449 encounters.

Patients had a mean age of 59.5 years (standard deviation: 18.0 ) and $48.9 \%$ were male. The baseline risk of dying during hospitalization was $4.8 \%$.

Table 1 describes patients by diabetes and peak serum glucose status. Patients with diagnosed diabetes were more likely to be older and male. Patients with elevated peak serum glucose $(>200 \mathrm{mg} / \mathrm{dL} ;>11.1$ $\mathrm{mmol} / \mathrm{L}$ ) were younger than the diagnosed diabetes group and had a higher baseline probability of inhospital death $(9.4 \%, 95 \%$ CI: 9.0-9.7). Patients in these 2 groups had more inpatient admissions within the previous 6 months compared to the groups with other peak serum glucose values.

Of the 5082 patients classified with a peak serum glucose measurement $>200 \mathrm{mg} / \mathrm{dL}$ (11.1 mmol/L), $15 \%$ had 2 and $8 \%$ had more than 2 serum glucose measurements that exceeded this threshold. For the remaining patients (with 1 peak serum glucose measurement $>200 \mathrm{mg} / \mathrm{dL}$ ), $52 \%$ had additional serum glucose measurements over $140 \mathrm{mg} / \mathrm{dL}(7.8 \mathrm{mmol} / \mathrm{L})$.

Table 2 presents crude 1 - and 5 -year mortality and morbidity rates by patient group. The mortality rate is the percentage of patients who died within 1 year and 5 years of their index admission, with or without developing a CV complication. Morbidity rate describes the percentage who developed the complication among patients who survived after discharge or developed the complication prior to death within a 1year and 5-year period. During the 1-year follow-up period, the crude mortality rate among patients with elevated peak serum glucose was higher than all other groups $(25.4 \%$ vs $20.6 \%$ for diagnosed diabetes and $7.4 \%$ to $22.5 \%$ for other peak serum glucose levels; all $P<0.0001)$. For the 5-year follow-up, the gap between patients with elevated peak serum glucose and those with diagnosed diabetes lessened but remained significant $(45.1 \%$ vs $41.7 \% ; P<0.0001)$. In the 1-year follow-up, patients with diagnosed diabetes had significantly higher morbidity rates than all other groups except for AMI. The difference in the rate of AMI for diagnosed diabetes and patients with elevated glucose was not statistically significant.

The 1-year adjusted hazard ratios for mortality and $\mathrm{CV}$ complications are presented in Figure 1. After adjustment for baseline demographics and clinical and hospital factors, having a peak serum glucose level above $200 \mathrm{mg} / \mathrm{dL}(11.1 \mathrm{mmol} / \mathrm{L})$ was an independent predictor of death. The mortality risk for this group in 
TABLE 1. Baseline Characteristics of The Ottawa Hospital Cohort $(\mathrm{N}=183,449)$ by Diabetes Status and Serum Glucose Level

\begin{tabular}{|c|c|c|c|c|c|c|}
\hline & \multirow[b]{2}{*}{$\begin{array}{c}\text { Diagnosed } \\
\text { Diabetes, } \\
\mathrm{n}=32,774,17.9 \%\end{array}$} & \multicolumn{5}{|c|}{ Serum Glucose Level, mg/dL } \\
\hline & & $\begin{array}{c}>200, n=5,082 \\
2.8 \%\end{array}$ & $\begin{array}{c}140-200 \\
\mathrm{n}=25,857,14.1 \%\end{array}$ & $\begin{array}{c}108-140, \\
n=38,741, \\
21.1 \%\end{array}$ & $\begin{array}{c}<108 \\
n=27,603 \\
15.0 \%\end{array}$ & $\begin{array}{c}\text { Unknown, } \\
n=53,392, \\
29.1 \%\end{array}$ \\
\hline Age, y, mean (SD) & $65.8(14.1)$ & $64.3(17.0)^{*}$ & $63.9(17.3)^{*}$ & $60.9(18.6)^{*}$ & $54.3(19.7)^{\star}$ & $54.8(16.9)^{\star}$ \\
\hline Risk-adjusted mortality at admission, mean (SD) & $0.08(0.12)$ & $0.09(0.12)^{*}$ & $0.07(0.10)^{*}$ & $0.05(0.09)^{*}$ & $0.04(0.07)^{*}$ & $0.01(0.04)^{*}$ \\
\hline Sex, male, no. (\%) & $18,200(55.5)$ & $2,610(51.4)^{\star}$ & $13,477(52.1)^{\star}$ & $19,495(50.3)^{*}$ & $12,907(46.8)^{*}$ & $22,951(43.0)^{\star}$ \\
\hline \multicolumn{7}{|l|}{ №. of previous inpatient admissions, 6 months (\%) } \\
\hline 0 & $22,780(69.5)$ & $3,576(70.4)$ & $19,118(73.9)^{*}$ & $28,827(74.4)^{*}$ & $20,354(73.7)^{\star}$ & $43,317(81.1)^{\star}$ \\
\hline 1 & $6,470(19.7)$ & $962(18.9)$ & $4,484(17.3)^{\star}$ & $6,526(16.9)^{\star}$ & $4,744(17.2)^{*}$ & $7,360(13.8)^{\star}$ \\
\hline $2+$ & $3,524(10.8)$ & $544(10.7)$ & $2,255(8.7)^{\star}$ & $3,388(9.1)^{\star}$ & $2,505(9.1)^{\star}$ & $2,715(5.1)^{\star}$ \\
\hline \multicolumn{7}{|l|}{ №. of previous emergency admissions ( 6 months) (\%) } \\
\hline 0 & $15,518(47.4)$ & $2,638(51.9)^{\star}$ & $12,681(49.0)^{*}$ & $16,584(42.8)^{*}$ & $13,039(47.2)$ & $43,112(80.8)^{\star}$ \\
\hline 1 & $9,665(29.5)$ & $1,490(29.3)^{*}$ & $8,339(32.3)^{*}$ & $13,829(35.7)^{*}$ & $8,709(31.6)$ & $6,533(12.2)^{*}$ \\
\hline $2+$ & $7,591(23.2)$ & $954(18.8)^{*}$ & $4,837(18.7)^{\star}$ & $8,328(21.5)^{\star}$ & $5,855(21.2)$ & $3,747(7.0)^{*}$ \\
\hline Emergency admission at index hospitalization (\%) & $25,420(77.6)$ & $4,178(82.2)^{*}$ & $20,284(78.5)^{\star}$ & $33,282(85.9)^{*}$ & $23,598(85.5)^{\star}$ & $15,039(28.2)^{\star}$ \\
\hline Length of stay, d (mean/SD) & $12.4(22.3)$ & $15.8(30.0)^{*}$ & $11.9(17.1)^{*}$ & $8.5(12.2)^{*}$ & $6.3(9.5)^{*}$ & $3.4(4.9)^{*}$ \\
\hline \multicolumn{7}{|l|}{ Disease at index hospitalization (\%) } \\
\hline PVD & $2,783(8.5)$ & $257(5.1)^{\star}$ & $1,387(5.4)^{\star}$ & $1,372(3.5)^{\star}$ & $908(3.3)^{\star}$ & $965(1.8)^{\star}$ \\
\hline Pneumonia & $3,308(10.1)$ & $658(13.0)^{*}$ & $2,577(10.0)$ & $2,809(7.3)^{\star}$ & $1,220(4.4)^{*}$ & $399(0.8)^{*}$ \\
\hline UTI & $3,549(10.8)$ & $665(13.1)^{*}$ & $2,660(10.3)^{\star}$ & $3,336(8.6)^{\star}$ & $1,559(5.7)^{\star}$ & $929(1.7)^{\star}$ \\
\hline HWD & $8,957(27.3)$ & $1,086(21.4)^{\star}$ & $4,714(18.2)^{*}$ & $5,306(13.7)^{\star}$ & $3,100(11.2)^{\star}$ & $2,842(5.3)^{*}$ \\
\hline Hypertension & $10,864(33.2)$ & $1,115(21.9)^{\star}$ & $5,155(19.9)^{*}$ & $5,880(15.2)^{\star}$ & $3,045(11.0)^{*}$ & $3,211(6.0)^{*}$ \\
\hline Arrhythmia & $5,010(15.3)$ & $823(16.2)$ & $3,768(14.6)^{*}$ & $3,718(9.6)^{\star}$ & $2,046(7.4)^{*}$ & $1,172(2.2)^{*}$ \\
\hline CHF & $570(17.5)$ & $763(15.0)^{*}$ & $2,761(10.7)^{\star}$ & $2,449(6.3)^{\star}$ & $1,121(4.1)^{*}$ & $386(0.7)^{*}$ \\
\hline Diagnoses of diabetes in the next year (\%) & - & $126(2.5)$ & $368(1.4)$ & $370(1.0)$ & $215(0.8)$ & $540(1.0)$ \\
\hline Diagnoses of diabetes in the next 5 years (\%) & - & $310(6.1)$ & $981(3.8)$ & $916(2.4)$ & $434(1.7)$ & $1,317(2.5)$ \\
\hline \multirow[t]{3}{*}{ First 3 most common primary discharge codes } & AHD I251 (5.7\%), & AHD 1251 (2.7\%), & AHD 1251 (4.0\%), & AHD I251 (1.6\%), & AHD $1251(1.8 \%)$ & OA M171 (2.3\%), \\
\hline & HF I50 (3.1\%), & COPD J441 (2.1\%), & Pneumonia J189 (1.6\%), & Pneumonia J189 (1.5\%), & AA K359 (1.7\%), & AHD I251 (2.2\%), \\
\hline & NSTEMI I214 (1.6\%) & HF I50 (2.0\%) & HF I50 (1.5\%) & CA D700 (1.3\%) & UA I200 (1.2\%) & OA M170 (1.7\%) \\
\hline
\end{tabular}

NOTE: Abbreviations: AA, acute appendicitis; $\mathrm{AHD}$, atherosclerotic heart disease; $\mathrm{CA}$, congenital agranulocytosis; $\mathrm{CHF}$, congestive heart failure; $\mathrm{COPD}$, chronic obstructive pulmonary disease; HF, heart failure; IHD, ischemic heart disease; NSTEMI, non-ST-elevation myocardial infarction; OA, osteoarthritis; PVD, peripheral vascular disease; SD, standard deviation; UA, unstable angina; UTI, urinary tract infection.

*Statistically significant difference $(P<0.05)$ from diagnosed diabetes group.

TABLE 2. Crude Mortality and Morbidity Rates

\begin{tabular}{|c|c|c|c|c|c|c|}
\hline & \multirow{2}{*}{$\begin{array}{c}\text { Diagnosed Diabetes, } \\
n=32,774\end{array}$} & \multicolumn{5}{|c|}{ Serum Glucose Level (mg/dL) } \\
\hline & & $>200, n=5,082$ & $140-200, n=25,857$ & $108-140, n=38,741$ & $<108, n=27,603$ & Unknown, $n=53,392$ \\
\hline \multicolumn{7}{|c|}{ All-cause death } \\
\hline 1 year & $6,762(20.6)$ & $1,293(25.4)^{*}$ & $5,309(20.5)$ & $7,212(18.6)^{*}$ & $4,178(15)^{*}$ & $3,969(7.4)^{*}$ \\
\hline 5 years & $13,659(41.7)$ & $2,292(45.1)^{*}$ & $9,871(38.2)^{*}$ & $13,256(34.2)^{\star}$ & $7,606(27.6)^{*}$ & $98,614(16.1)^{*}$ \\
\hline \multicolumn{7}{|l|}{ AMl } \\
\hline 1 year & $728(2.8)$ & $104(2.7)$ & $326(1.6)^{*}$ & $386(1.2)^{\star}$ & $172(0.7)^{\star}$ & $218(0.4)^{\star}$ \\
\hline 5 years & $1,687(8.4)$ & $182(6.3)^{*}$ & $687(4.2)^{*}$ & $837(3.2)^{*}$ & $436(2.2)^{*}$ & $633(1.4)^{*}$ \\
\hline \multicolumn{7}{|l|}{ CVD } \\
\hline 1 year & $582(2.2)$ & $71(1.9)$ & $306(1.5)^{*}$ & $403(1.3)^{*}$ & $251(1.1)^{*}$ & $195(0.4)^{*}$ \\
\hline 5 years & $1,153(5.8)$ & $143(5.0)$ & $623(3.8)^{*}$ & $872(3.4)^{*}$ & $470(2.3)^{*}$ & $534(1 .)^{*}$ \\
\hline \multicolumn{7}{|l|}{ CHF } \\
\hline 1 year & $2,260(8.4)$ & $245(6.3)^{*}$ & $870(4.2)^{*}$ & $1,023(3.2)^{*}$ & $516(2.2)^{*}$ & $362(0.7)^{*}$ \\
\hline 5 years & $3,830(17.7)$ & $395(12.9)^{*}$ & $1,529(9.0)^{*}$ & $1,802(6.8)^{*}$ & $960(4.7)^{*}$ & $884(2.0)^{*}$ \\
\hline \multicolumn{7}{|l|}{ PVD } \\
\hline 1 year & $795(3.0)$ & $24(0.6)^{*}$ & $114(0.6)^{*}$ & $154(0.5)^{*}$ & $97(0.4)^{*}$ & $141(0.3)^{*}$ \\
\hline 5 years & $1,547(7.8)$ & $50(1.8)^{*}$ & $220(1.4)^{*}$ & $275(1.1)^{*}$ & $192(1.0)^{*}$ & $295(0.7)^{*}$ \\
\hline \multicolumn{7}{|l|}{ ESRD } \\
\hline 1 year & $953(3.6)$ & $104(2.7)^{*}$ & $403(1.9)^{*}$ & $457(1.4)^{*}$ & $311(1.3)^{*}$ & $329(0.7)^{*}$ \\
\hline 5 years & $1,938(9.5)$ & $183(6.3)^{*}$ & $807(4.9)^{*}$ & $995(3.8)^{*}$ & $611(3.0)^{*}$ & $771(1.7)^{*}$ \\
\hline
\end{tabular}

NOTE: Data are presented as absolute values (percentages). Abbreviations: AMI, acute myocardial infarction; CHF, congestive heart failure; CVD, cardiovascular disease; ESRD, end stage renal disease; PVD, peripheral vascular disease.

*Statistically significant difference $(P<0.05)$ from diagnosed diabetes group. 


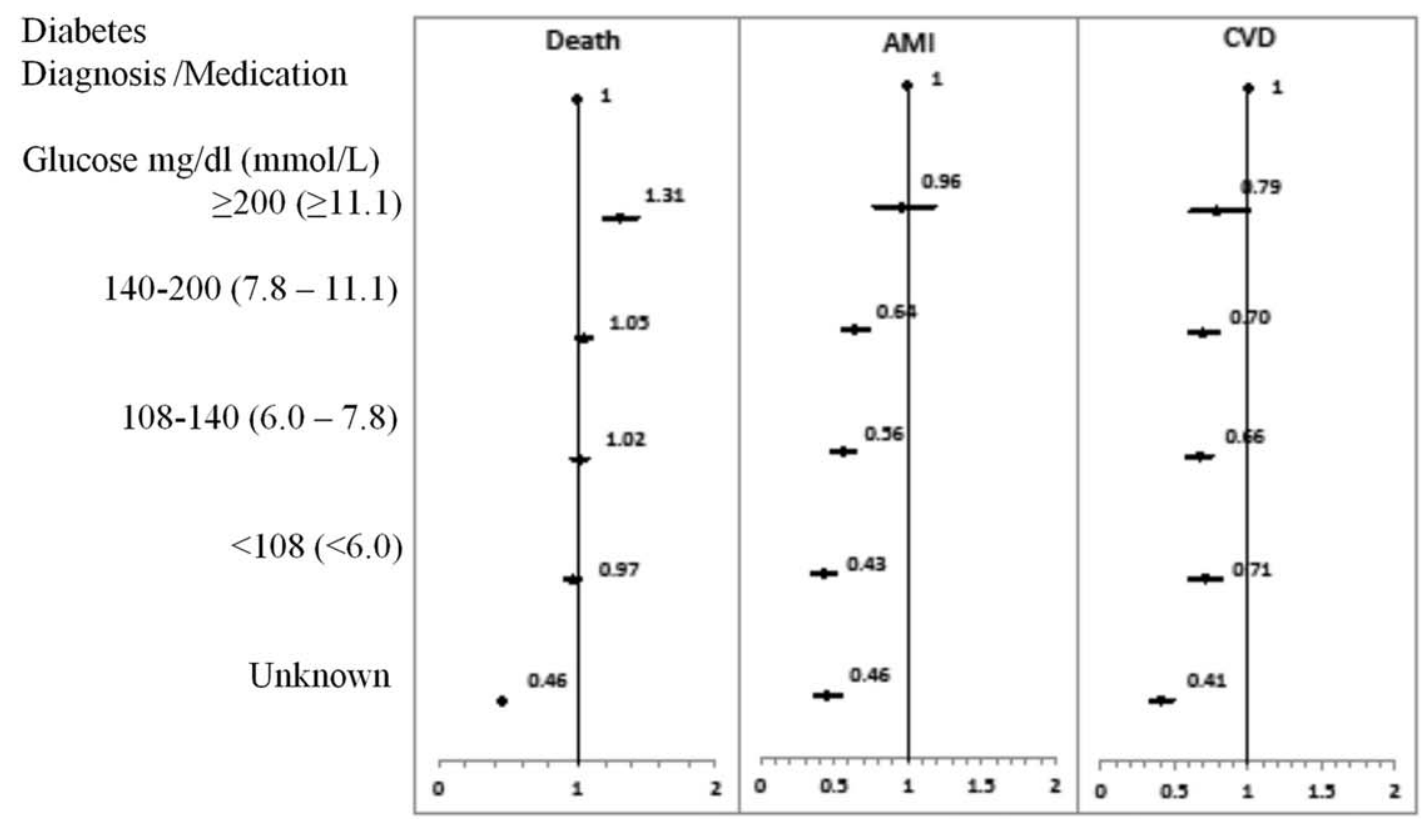

\section{Diabetes \\ Diagnosis /Medication}

Glucose mg/dl (mmol/L) $\geq 200(\geq 11.1)$

$140-200(7.8-11.1)$

$108-140(6.0-7.8)$

$<108(<6.0)$

Unknown
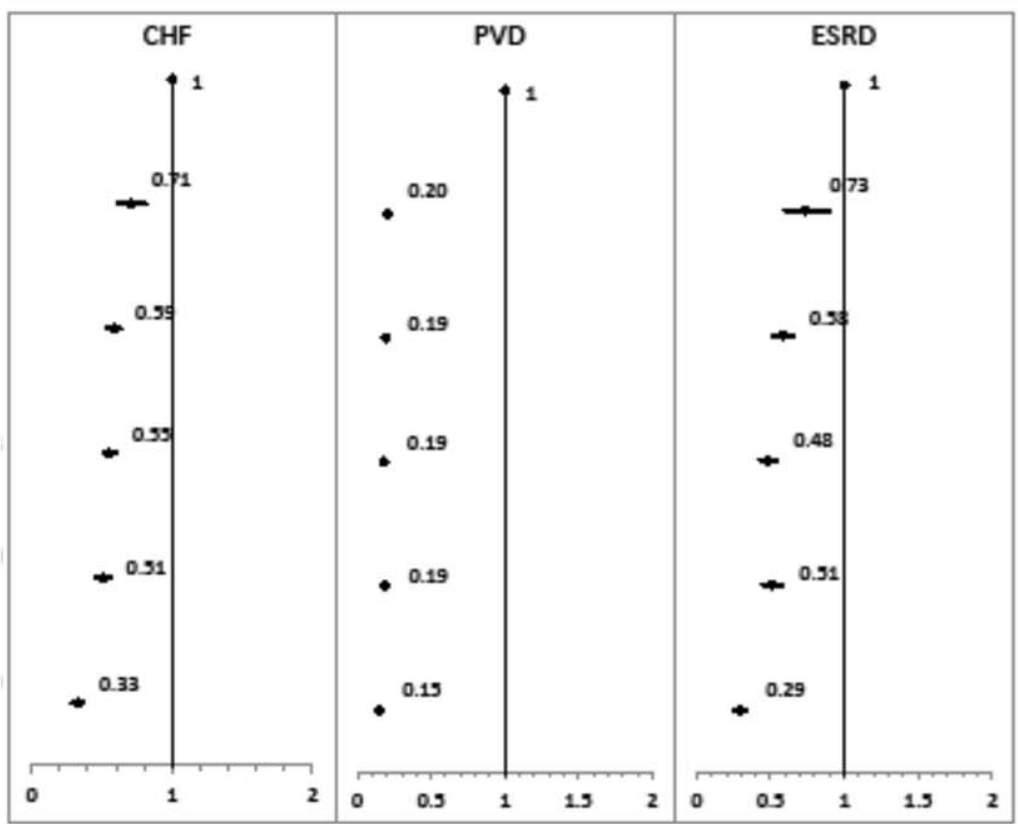

Adjusted for sex, Charlson index and previous hospitalizations

FIG. 1. Adjusted 1-year hazard ratios for death and diabetes complications. Abbreviations: AMI, acute myocardial infarction; CHF, congestive heart failure; CVD, cardiovascular disease; ESRD, end-stage renal disease; PVD, peripheral vascular disease.

the year following discharge was $31 \%$ higher than patients with diagnosed diabetes (adjusted HR: 1.31, 95\% CI: 1.20-1.43). There was no mortality risk difference between the group with diagnosed diabetes and the lower peak serum glucose levels (adjusted HR: 1.05, 95\% CI: 0.99-1.11), 108-160 mg/dL (6.0-7.8 mmol/L) (adjusted HR: 1.02, 95\% CI: 0.97-1.07); and $<108 \mathrm{mg} / \mathrm{dL}(<6.0 \mathrm{mmol} / \mathrm{L}$ ) (adjusted HR: 0.97, 95\% CI: 0.92-1.03). Adjusted HRs, with $95 \%$ CIs and $P$ values for 5-year follow-up are presented in Figure 2.

Adjusted hazard ratios for morbidity showed a different pattern. Patients with diagnosed diabetes had a higher risk of developing complications in the year following discharge from the hospital (Figure 1). After adjusting for potential confounders, we found that patients with diagnosed diabetes had the same risk of AMI and CVD as patients with peak serum glucose levels above $200 \mathrm{mg} / \mathrm{dL}$ (11.1 mmol/L) (AMI, adjusted HR: 0.96 ; 95\% CI: 0.78-1.18; CVD, adjusted HR: 0.79 ; $95 \%$ CI: $0.61-1.00$ ) but a $36 \%$ to $57 \%$ higher AMI risk and $29 \%$ to $34 \%$ higher CVD risk than other peak serum glucose level groups. Compared to all peak serum glucose groups, patients with diagnosed diabetes had a significantly higher risk of 
Diabetes

Diagnosis /Medication

Glucose $\mathrm{mg} / \mathrm{dl}(\mathrm{mmol} / \mathrm{L})$ $\geq 200(\geq 11.1)$

$140-200(7.8-11.1)$

$108-140(6.0-7.8)$

$<108(<6.0)$

Unknown

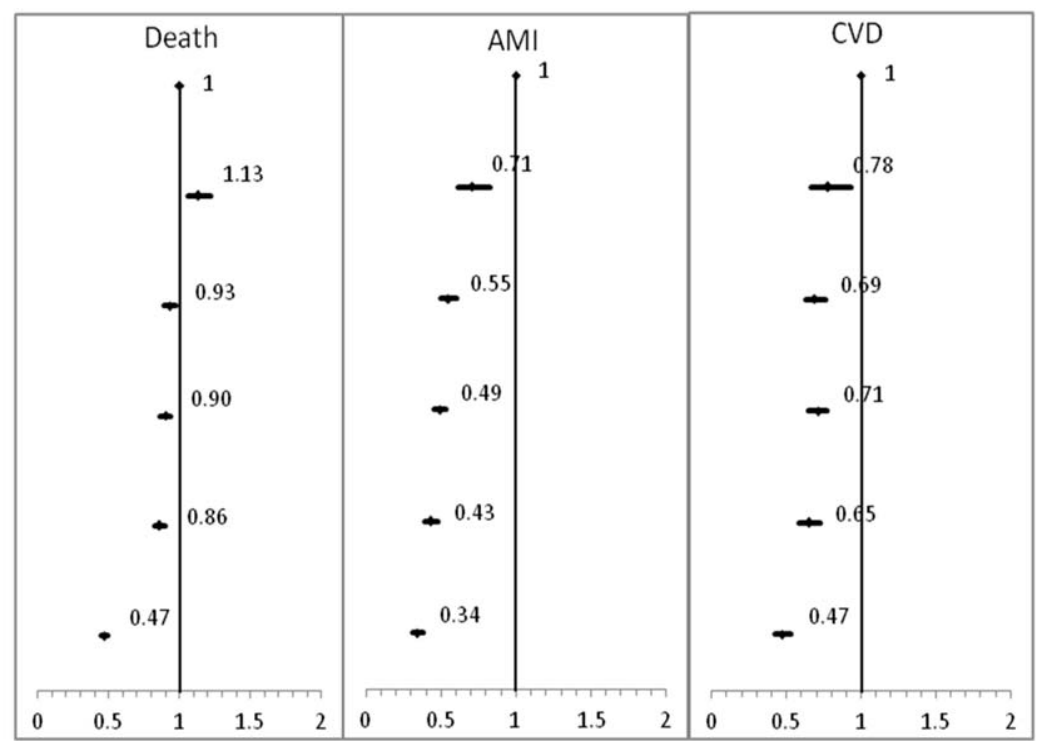

Diabetes

Diagnosis /Medication

Glucose $\mathrm{mg} / \mathrm{dl}(\mathrm{mmol} / \mathrm{L})$ $\geq 200(\geq 11.1)$

$140-200(7.8-11.1)$

$108-140(6.0-7.8)$

$<108(<6.0)$
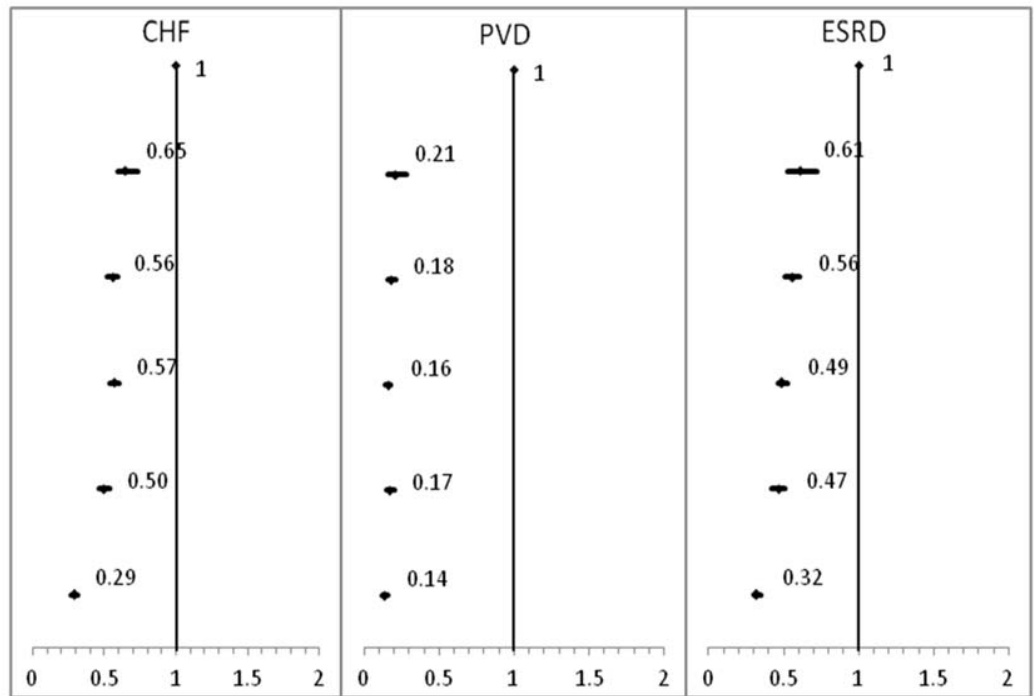

Adjusted for sex, Charlson index and previous hospitalizations

FIG. 2. Adjusted 5-year hazard ratios for death and diabetes complications. Abbreviations: AMI, acute myocardial infarction; CHF, congestive heart failure; CVD, cardiovascular disease; ESRD, end-stage renal disease; PVD, peripheral vascular disease.

developing CHF, PVD, and ESRD (Figure 1). Similarly, the 5-year risk of AMI, CVD, CHF, PVD, and ESRD was higher for patients with diagnosed diabetes compared to all peak serum glucose groups (Figure 2).

\section{CONCLUSION/DISCUSSION}

The study found that in-hospital hyperglycemia was a strong predictor of mortality at 1 - and 5-years followup, even after adjustment using well-established and discriminating comorbidity measures. Extreme inhospital hyperglycemia was a stronger predictor of mortality than diagnosed diabetes.

Previous studies have shown that diabetes and stress hyperglycemia among hospitalized patients are important markers for poor clinical outcomes and inhospital mortality. ${ }^{1,2,4}$ This study indicates that hospitalized patients with extreme hyperglycemia (peak serum glucose $>200 \mathrm{mg} / \mathrm{dL}$ ) were at a high risk of death for at least 5 years following discharge.

These findings, based on a large general sample of hospitalized patients, indicate that the extreme elevations in peak serum glucose convey a risk for all hospitalized patients, not only those with critical illness. ${ }^{3,18}$ Hyperglycemia appears to be an independent indicator of mortality risk and should be evaluated as a potential component within risk prediction tools. Further study is required to determine mechanisms for this risk association to identify what therapies, if any, might be used to minimize this risk.

The diagnosed diabetes group, which likely included some patients who also had extreme in-hospital hyperglycemia, had a lower 1-year risk of death than patients with hyperglycemia who did not have diabetes diagnosis. This may be an indication of the 
protective effect of blood glucose control, because patients with diabetes are more likely to receive therapy for hyperglycemia during and after hospitalization.

Classification of patients by several serum glucose levels (as opposed to a dichotomous classification, where hyperglycemia is either present or absent), showed that hyperglycemia constitutes a graded risk ${ }^{5}$ for almost all outcomes examined, particularly mortality, AMI, and CVD.

Previous studies indicate that diabetes is observed in only $23 \%$ to $35 \%$ of hospitalized patients with hyperglycemia. ${ }^{18,19}$ We would expect higher risk for CV complications for patients with elevated glucose if the proportion of these patients who had undiagnosed diabetes was higher than the proportion estimated in the literature. However, we observed lower risk of CV complications (especially PVD and ESRD) for the elevated glucose group in the 1 and 5 years following discharge. In-hospital hyperglycemia is not equivalent to undiagnosed diabetes.

There are several potential limitations in this study. The first is our method for ascertaining CV complications. Diverse disease definitions are used in medical literature, and similar studies using different definitions may yield different results, though we would not expect to find a wide range of variation. Second, our study did not include information about severity of diabetes and the persistence of elevated glucose; if available, this knowledge may provide better insight into patient experiences, especially in long-term follow-up. Third, results are also limited by the absence of data on cause of death, a potentially helpful means of identifying posthospitalization difficulties experienced by patients with hyperglycemia. Fourth, this study mainly compares experiences of patients with elevated peak serum glucose level to diabetes patients; it would be worthwhile to explore the impact of lower gradations of glucose levels.

We would like to emphasize that we did not confirm diabetes diagnosis following discharge for patients with hyperglycemia. However, we did not observe a high rate of complications at 5-year followup, particularly for ESRD. This may be because most patients with in-hospital elevated glucose had early diabetes or transient hyperglycemia and therefore lower risk of long-term diabetes-specific consultations.

Hyperglycemia is an important independent indicator, carrying a greater risk for 1 - and 5-year mortality than diagnosed diabetes. However, it is unclear whether hospitalized patients with elevated peak serum glucose have early diabetes or their hyperglycemia reflects hospital stress or another comorbidity concept.

\section{Acknowledgements}

The authors are grateful to Amy Zierler and Allison Whalen O'Connor for their editorial assistance. This study was supported by the Institute for Clinical Evaluative Sciences (ICES), which is funded by an annual grant from the Ontario Ministry of Health and Long-Term Care (MOHLTC). The opinions, results and conclusions reported in this paper are those of the authors and are independent from the funding sources. No endorsement by ICES or the Ontario MOHLTC is intended or should be inferred.

Disclosure: Nothing to report.

\section{References}

1. Clement S, Braithwaite SS, Magee MF, et al. Management of diabetes and hyperglycemia in hospitals. Diabetes Care. 2004;27(2):553-591.

2. Moghissi ES, Korytkowski MT, DiNardo M, et al. American Association of Clinical Endocrinologists and American Diabetes Association consensus statement on inpatient glycemic control. Diabetes Care. 2009;32(6):1119-1131.

3. The NICE-SUGAR Study Investigators. Intensive versus conventional glucose control in critically ill patients. N Engl J Med. 2009;360(13): 1283-1297.

4. Umpierrez GE, Isaacs SD, Bazargan N, You X, Thaler LM, Kitabchi AE. Hyperglycemia: an independent marker of in-hospital mortality in patients with undiagnosed diabetes. J Clin Endocrinol Metab. 2002; 87(3):978-982.

5. Falciglia M1, Freyberg RW, Almenoff PL, D’Alessio DA, Render ML. Hyperglycemia-related mortality in critically ill patients varies with admission diagnosis. Crit Care Med. 2009;37(12):3001-3009.

6. Lleva RR, Inzucchi SE. Hospital management of hyperglycemia. Curr Opin Endocrinol Diabetes Obes. 2011;18(2):110-118.

7. Saposnik G, Kapral MK, Liu Y, et al. IScore: a risk score to predict death early after hospitalization for an acute ischemic stroke. Circulation. 2011;123(7):739-749.

8. Karunakar MA, Staples KS. Does Stress-induced hyperglycemia increase the risk of perioperative infectious complications in orthopaedic trauma patients? J Orthop Trauma. 2010;24(12):752-756.

9. Mechanick JI. Metabolic mechanisms of stress hyperglycemia. J Parenter Enteral Nutr. 2006;30(2):157-163.

10. Tu JV, Naylor CD, Austin P. Temporal changes in the outcomes of acute myocardial infarction in Ontario, 1992-1996. CMAJ. 1999; 161(10):1257-1261.

11. Ho ML, Lawrence N, van Walraven C, et al. The accuracy of using integrated electronic health care data to identify patients with undiagnosed diabetes mellitus. J Eval Clin Pract. 2012;18(3):606-611.

12. American Diabetes Association. Diagnosis and classification of diabetes mellitus. Diabetes Care. 2006;29(suppl 1):s43-s48.

13. Pintilie M. Dealing with competing risks: testing covariates and calculating sample size. Stat Med. 2002;21(22):3317-3324.

14. Melberg T, Nygard OK, Kuiper KK, Nordrehaug JE. Competing risk analysis of events 10 years after revascularization. Scand Cardiovasc J. 2010;44(5):279-288.

15. Escobar GJ, Greene JD, Scheirer P, Gardner MN, Draper D, Kipnis P. Risk-adjusting hospital inpatient mortality using automated inpatient, outpatient, and laboratory databases. Med Care. 2008;46(3):232239.

16. van Walraven C, Escobar GJ, Greene JD, Forster AJ. The Kaiser Permanente inpatient risk adjustment methodology was valid in an external patient population. J Clin Epidemiol. 2010;63(7):798-803.

17. Schneeweiss S, Wang PS, Avorn J, Glynn RJ. Improved comorbidity adjustment for predicting mortality in medicare populations. Health Serv Res. 2003;38(4):1103-1120.

18. Norhammar A, Tenerz A, Nilsson G, et al. Glucose metabolism in patients with acute myocardial infarction and no previous diagnosis of diabetes mellitus: a prospective study. Lancet. 2002;359(9324): 2140-2144.

19. Matz K, Keresztes K, Tatschl C, et al. Disorders of glucose metabolism in acute stroke patients: an underrecognized problem. Diabetes Care. 2006;29(4):792-797. 